\title{
Prime Labeling for Some Cycle Related Graphs
}

\author{
S K Vaidya (Corresponding author) \\ Department of Mathematics, Saurashtra University \\ Rajkot 360 005, Gujarat, India \\ E-mail: samirkvaidya@yahoo.co.in \\ K K Kanani \\ Mathematics Department, L E College, \\ Morbi 363 642, Gujarat, India \\ E-mail:kananikkk@yahoo.co.in
}

\begin{abstract}
Here we investigate prime labeling for some cycle related graphs. We also discuss prime labeling in the context of some graph operations namely fusion, duplication and vertex switching in cycle $C_{n}$.
\end{abstract}

Keywords: Prime labeling, Duplication, Vertex switching

\section{Introduction}

We begin with finite, connected and undirected graph $G=(V(G), E(G))$ without loops and multiple edges. Here elements of sets $V(G)$ and $E(G)$ are known as vertices and edges respectively. In the present work $C_{n}$ denotes cycle with $n$ vertices and $N(v)$ denotes the set of all neighboring vertices of $v$. For all other terminology and notations in graph theory we follow (Gross, J., 1999) where as for number theory we follow (Niven, I., 1972). We will give brief summary of definitions which are useful for the present investigations.

Definition 1.1 If the vertices of the graph are assigned values subject to certain conditions is known as graph labeling. Enough literature is available in printed as well as in electronic form on different types of graph labeling and more than 1000 research papers have been published so far in past four decades. A current survey of various graph labeling problems can be found in (Gallian, J., 2009).

Following three are the common features of any graph labeling problem.

(1) a set of numbers from which vertex labels are assigned;

(2) a rule that assigns a value to each edge;

(3) a condition that these values must satisfy.

The present work is targeted to discuss one such labeling known as prime labeling defined as follows.

\section{Definition 1.2}

Let $G=(V(G), E(G))$ be a graph with $p$ vertices. A bijection $f: V(G) \rightarrow\{1,2, \ldots, p\}$ is called a prime labeling if for each edge $e=u v, \operatorname{gcd}(f(u), f(v))=1$. A graph which admits prime labeling is called a prime graph.

The notion of a prime labeling was introduced by Roger Entringer and was discussed in a paper by (Tout, A,1982, p.365368). Many researchers have studied the prime graphs. For e.g. in (Fu, H., 1994, p.181-186) have proved that path $P_{n}$ on $n$ vertices is a prime graph. In (Deretsky, T., 1991, p.359-369) have proved that the cycle $C_{n}$ on $n$ vertices is a prime graph. In (Lee, S., 1988, p.59-67) have proved that wheel $W_{n}$ is a prime graph if and only if $n$ is even. Around 1980 Roger Etringer conjectured that All trees have prime labeling which is not settled till today. The prime labeling for planar grid is investigated by (Sundaram, M., 2006, p.205-209).

Definition 1.3 An independent set of vertices in a graph $G$ is a set of mutually non-adjacent vertices.

Definition 1.4 The independence number of a graph $G$ is the maximum cardinality of an independent set of vertices. It is denoted by ind $(G)$ or $\alpha(G)$.

Definition 1.5 Let $u$ and $v$ be two distinct vertices of a graph $G$. A new graph $G_{1}$ is constructed by identifying(fusing) two vertices $u$ and $v$ by a single vertex $x$ is such that every edge which was incident with either $u$ or $v$ in $G$ is now incident with $x$ in $G_{1}$.

Definition 1.6 Duplication of a vertex $v_{k}$ of graph $G$ produces a new graph $G_{1}$ by adding a vertex $v_{k}^{\prime}$ with $N\left(v_{k}^{\prime}\right)=N\left(v_{k}\right)$. In other words a vertex $v_{k}^{\prime}$ is said to be duplication of $v_{k}$ if all the vertices which are adjacent to $v_{k}$ are now adjacent to $v_{k}^{\prime}$ 
also.

Definition 1.7 A vertex switching $G_{v}$ of a graph $G$ is obtained by taking a vertex $v$ of $G$, removing all the edges incident with $v$ and adding edges joining $v$ to every vertex which are not adjacent to $v$ in $G$.

Definition 1.8 (Shee, S.,\& Ho, Y., 1996, p.221-229) Let $G_{1}, G_{2}, \ldots, G_{n}, n \geq 2$ be $n$ copies of a fixed graph $G$. The graph obtained by adding an edge between $G_{i}$ and $G_{i+1}$ for $i=1,2, \ldots n-1$ is called the path union of $G$.

In the present work we prove that the graphs obtained by identifying any two vertices, duplicating arbitrary vertex and switching of any vertex in cycle $C_{n}$ admit prime labeling. We also prove that the graph obtained by path union of cycle $C_{n}$ is a prime graph except for odd $n$. In addition to this we show that the graph obtained by joining two copies of cycle $C_{n}$ by a path of arbitrary length is a prime graph except $n$ and $k$ both are odd.

\section{Main Results}

Theorem 2.1 The graph obtained by identifying any two vertices $v_{i}$ and $v_{j}$ (where $d\left(v_{i}, v_{j}\right) \geq 3$ ) of cycle $C_{n}$ is a prime graph.

Proof: Let $C_{n}$ be the cycle with vertices $v_{1}, v_{2}, \ldots, v_{n}$ and the vertex $v_{1}$ be fused with $v_{m}$ where $m \leq\lceil n / 2\rceil$. Denote the resultant graph as $G$. Here we note that $|V(G)|=n-1$. It is obvious that identifying two vertices of cycle $C_{n}$ produces connected graph which includes two edge disjoint cycles $C_{m-1}$ and $C_{n-m+1}$.

To define labeling $f: V(G) \rightarrow\{1,2, \ldots, n-1\}$ following cases are to be considered.

Case 1: If $n \equiv 0(\bmod 2)$

$$
\begin{gathered}
f\left(v_{i}\right)=i ; 1 \leq i<m \\
f\left(v_{i}\right)=i-1 ; m+1 \leq i \leq n
\end{gathered}
$$

Case 2: If $n \equiv 1(\bmod 2)$

$$
\begin{gathered}
f\left(v_{i}\right)=i ; 1 \leq i<m \\
f\left(v_{i}\right)=i-1 ; m+1 \leq i \leq n
\end{gathered}
$$

In each case described above the graph under consideration admits prime labeling i.e. $G$ is a prime graph.

Remark 2.2 (i)when $m>\left\lceil\frac{n}{2}\right\rceil$ identifying two vertices will repeat all the graphs which are already considered earlier for $m \leq\left\lceil\frac{n}{2}\right\rceil$.

(ii)when $d\left(v_{i}, v_{j}\right)<3$ then fusion yields a graph which is not simple and it is not desirable for prime labeling.

Illustration 2.3 Consider a graph $G$ obtained by identifying the vertex $v_{1}$ with $v_{6}$ of cycle $C_{11}$. It is the case related to $n \equiv 1(\bmod 2)$. The labeling is as shown in Fig 1 .

Theorem 2.4 The graph obtained by duplicating arbitrary vertex of cycle $C_{n}$ is a prime graph.

Proof: Let $v_{k}$ be any arbitrary vertex of cycle $C_{n}, v_{k}^{\prime}$ be its duplicated vertex and $G$ be the graph resulted due to duplication of vertex $v_{k}$. Then $|V(G)|=n+1$. Define labeling $f: V(G) \rightarrow\{1,2, \ldots, n+1\}$ as follows.

$$
\begin{gathered}
f\left(v_{k}\right)=4 \\
f\left(v_{k}^{\prime}\right)=2 \\
f\left(v_{k+1}\right)=3 \\
f\left(v_{k+i}\right)=i+3 ; 1<i<n-1 \\
f\left(v_{k+n-1}\right)=1
\end{gathered}
$$

Then for any edge $e=v_{i} v_{j} \in G, \operatorname{gcd}\left(f\left(v_{i}\right), f\left(v_{j}\right)\right)=1$.

Thus function defined above provides prime labeling for graph $G$ i.e. graph $G$ under consideration is a prime graph.

Illustration 2.5 Consider a graph $G$ obtained by duplicating a vertex $v_{1}$ in cycle $C_{6}$. The labeling is as shown in Fig 2 .

Theorem 2.6 The switching of any vertex in cycle $C_{n}$ produces a prime graph.

Proof: Let $G=C_{n}$ and $v_{1}, v_{2}, \ldots, v_{n}$ be the successive vertices of $C_{n}$ and $G_{v}$ denotes the vertex switching of $G$ with respect to the vertex $v$. It is obvious that $\left|V\left(G_{v}\right)\right|=2 n-5$. Without loss of generality we initiate the labeling from $v_{1}$ and proceed in the clockwise direction.

Define labeling $f: V\left(G_{v}\right) \rightarrow\{1,2, \ldots, n\}$ as follows.

$$
f\left(v_{i}\right)=i ; 1 \leq i \leq n
$$


Then for any edge $e=v_{i} v_{j} \in G_{v}, g c d\left(f\left(v_{i}\right), f\left(v_{j}\right)\right)=1$.

Thus $f$ admits a prime labeling and consequently $G_{v}$ is a prime graph.

Illustration 2.7 Consider a graph $G$ obtained by switching the vertex $v_{1}$ of cycle $C_{9}$. The corresponding prime labeling is shown in Fig 3.

Theorem 2.8 The graph obtained by the path union of finite number of copies of cycle $C_{n}$ is a prime graph except for odd $n$.

Proof: Let $G$ be the path union of cycle $C_{n}$ and $G_{1}, G_{2}, \ldots, G_{k}$ be $k$ copies of the cycle $C_{n}$. We note that $|V(G)|=n k$. Let us denote the successive vertices of the graph $G_{i}$ by $u_{i 1}, u_{i 2}, \ldots, u_{i n}$. Let $e_{i}=u_{i 1} u_{(i+1) 1}$ be the edge joining $G_{i}$ and $G_{i+1}$ for $i=1,2, \ldots, k-1$.

To define labeling $f: V(G) \rightarrow\{1,2, \ldots, n k\}$ following cases are to be considered.

Case 1: If $n \equiv 0(\bmod 2)$

Subcase I: $k \equiv 0(\bmod 2)$

$$
f\left(u_{i j}\right)=(i-1) n+j ; 1 \leq i \leq k, 1 \leq j \leq n
$$

Subcase II: $k \equiv 1(\bmod 2)$

$$
f\left(u_{i j}\right)=(i-1) n+j ; 1 \leq i \leq k, 1 \leq j \leq n
$$

Here we note that $\operatorname{gcd}\left(u_{i 1}, u_{(i+1) 1}\right)=1$ for each $1 \leq i \leq k$ because

Case 2: If $n \equiv 1(\bmod 2)$

$$
\begin{aligned}
\operatorname{gcd}\left(u_{i 1}, u_{(i+1) 1}\right) & =\operatorname{gcd}(2 m i-(2 m-1), 2 m(i+1)-(2 m-1)), \text { where } 2 m=n \\
& =\operatorname{gcd}(2 m i-(2 m-1), 2 m(i+1)-(2 m-1)-(2 m i-(2 m-1))) \\
& =\operatorname{gcd}(2 m i-(2 m-1), 2 m) \\
& =\operatorname{gcd}(2 m i-(2 m-1)-2 m i, 2 m) \\
& =\operatorname{gcd}(-(2 m-1), 2 m) \\
& =\operatorname{gcd}((2 m-1), 2 m) \\
& =\operatorname{gcd}((2 m-1), 2 m-(2 m-1)) \\
& =\operatorname{gcd}(2 m-1,1) \\
& =1
\end{aligned}
$$

In this case $|\alpha(G)|<\lfloor n / 2\rfloor$ and as proved in(Fu, H., 1994, p.181-186) the graph is not a prime graph.

Thus we proved that the graph obtained by the path union of finite number of copies of cycle $C_{n}$ is a prime graph except for odd $n$.

Illustration 2.9 Consider a graph $G$ obtained by path union of three copies of the cycle $C_{10}$. It is the case related to $n \equiv 0(\bmod 2)$ and $k \equiv 1(\bmod 2)$. The prime labeling is as shown in Fig 4 .

Theorem 2.10 The graph obtained by joining two copies of cycle $C_{n}$ by a path $P_{k}$ is a prime graph except $n$ and $k$ both are odd.

Proof: Let $G$ be the graph obtained by joining two copies of cycle $C_{n}$ by a path $P_{k}$. We note that $|V(G)|=2 n+k-2$. Let $u_{1}, u_{2}, \ldots, u_{n}$ be the vertices of first copy of cycle $C_{n}$ and $v_{1}, v_{2}, \ldots, v_{n}$ be the vertices of second copy of cycle $C_{n}$. Let $w_{1}, w_{2}, \ldots, w_{k}$ be the vertices of path $P_{k}$ with $u_{1}=w_{1}$ and $v_{1}=w_{k}$.

To define labeling $f: V(G) \rightarrow\{1,2, \ldots, 2 n, 2 n+1, \ldots, 2 n+k-2\}$ following cases are to be considered.

Case 1: If $n \equiv 0(\bmod 2)$

Subcase I: $k \equiv 0(\bmod 2)$

$$
\begin{gathered}
f\left(u_{i}\right)=i ; 1 \leq i \leq n \\
f\left(v_{i}\right)=n+i ; 1 \leq i \leq n \\
f\left(w_{j}\right)=2 n+k-j ; 1<j<k
\end{gathered}
$$

Subcase II: $k \equiv 1(\bmod 2)$

$$
\begin{gathered}
f\left(u_{i}\right)=i ; 1 \leq i \leq n \\
f\left(v_{i}\right)=n+i ; 1 \leq i \leq n \\
f\left(w_{j}\right)=2 n+k-j ; 1<j<k
\end{gathered}
$$


Case 2: If $n \equiv 1(\bmod 2)$

Subcase I: $k \equiv 0(\bmod 2)$

$$
\begin{gathered}
f\left(u_{i}\right)=i ; 1 \leq i \leq n \\
f\left(v_{i}\right)=n+i+1 ; 1 \leq i \leq n \\
f\left(w_{2}\right)=n+1 \\
f\left(w_{j}\right)=2 n+k-j+1 ; 2<j<k
\end{gathered}
$$

Subcase II: $k \equiv 1(\bmod 2)$

In this case $|\alpha(G)|<\lfloor n / 2\rfloor$. Then as proved in (Fu, H., 1994, p.181-186) the graph is not a prime graph.

Thus we derived that the graph obtained by joining two copies of cycle $C_{n}$ by a path $P_{k}$ is a prime graph except $n$ and $k$ both are odd.

Illustration 2.11 Consider a graph $G$ obtained by joining two copies of the cycle $C_{10}$ by a path $P_{3}$. It is the case related to $n \equiv 0(\bmod 2)$ and $k \equiv 1(\bmod 2)$. The labeling is as shown in Fig 5 .

\section{Concluding Remarks}

Labeled graph is the topic of current interest due to its diversified applications. We investigate five new results on prime labeling. It is an effort to relate the prime labeling and some graph operations. This approach is novel as it provides prime labeling for the larger graph resulted due to certain graph operations on a given graph.

\section{Open problems}

(1) It is possible to investigate similar results for other graph families.

(2) There is a scope to derive similar results corresponding to different graph labeling techniques.

\section{Acknowledgement}

The authors are thankful to the anonymous referee for useful suggestions and valuable comments.

\section{References}

Deretsky, T., Lee, S. M. \& Mitchem, J. (1991). On vertex prime labeling of graphs in Graph Theory, Combinatorics and Applications Proceedings 6th International Conference Theory and Applications of Graphs, Ed. J Alavi, G Chartrand, O. Oellerman and A. Schwenk, Vol 1, 359-369.

Fu, H. L. \& Huang, K. C. (1994). On prime labelings. Discrete Math., 127, 181-186.

Gallian, J. A. (2009). A dynamic survey of graph labeling. The Electronic Journal of Combinatorics, 16, \#DS 6 .

Gross, J. , \& Yellen, J. (1999). Graph Theory and Its Applications, CRC Press.

Lee, S. M., Wui, I. \& Yeh, J. (1988). On the amalgamation of prime graphs. Bull. Malaysian Math. Soc.(Second series), 11, 59-67.

Niven, I. \& Zuckerman, H. (1972). An Introduction to the Theory of numbers, Wiley Eastern, New Delhi.

Shee, S. C. \& Ho, Y. S. (1996). The Cordiality of Path-union of n Copies of a Graph, Discrete Math, 151, 221-229.

Sundaram, M., Ponraj. R. \& Somasundaram, S. (2006). On a prime labeling conjecture. Ars Combinatoria, 79, 205-209. Tout, A., Dabboucy, A. N. \& Howalla, K. (1982). Prime labeling of Graphs. Nat. Acad. Sci. Letters, 11, 365-368.

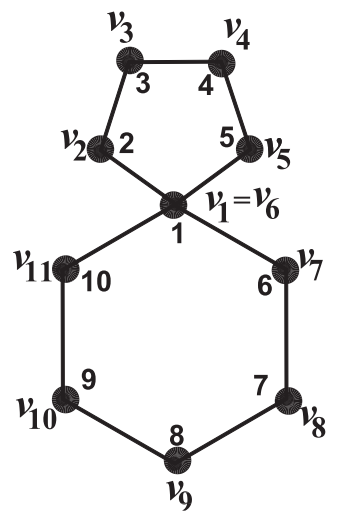

Figure 1. Fusion of $v_{1}$ with $v_{6}$ in $C_{11}$ and Prime labeling 


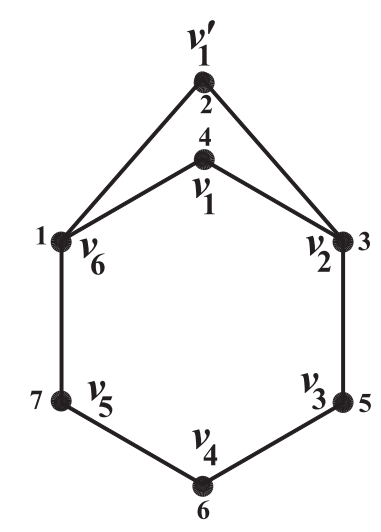

Figure 2. Vertex duplication in $C_{6}$ and Prime labeling

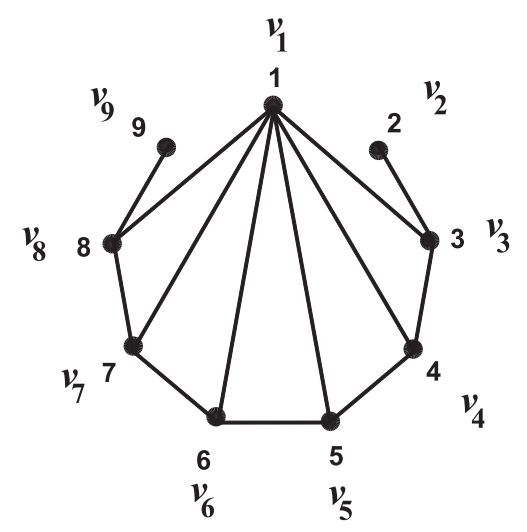

Figure 3. Vertex switching in $C_{9}$ and Prime labeling

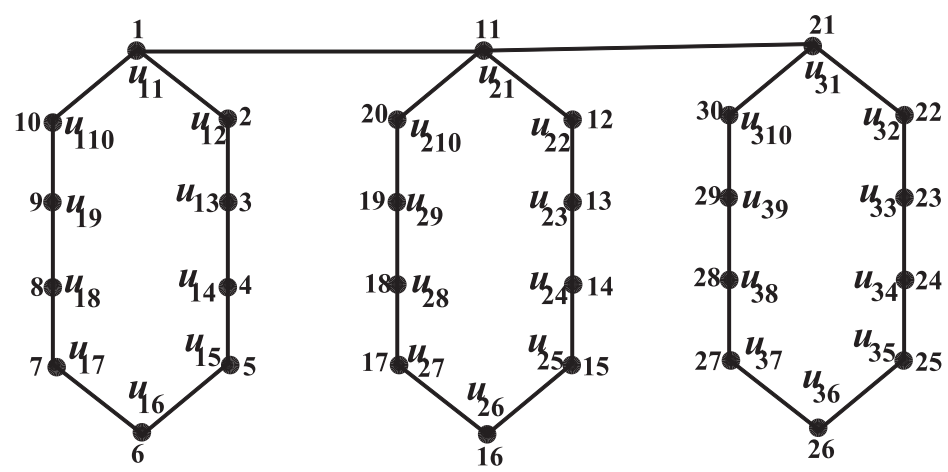

Figure 4. Path union of $C_{10}$ and Prime labeling 


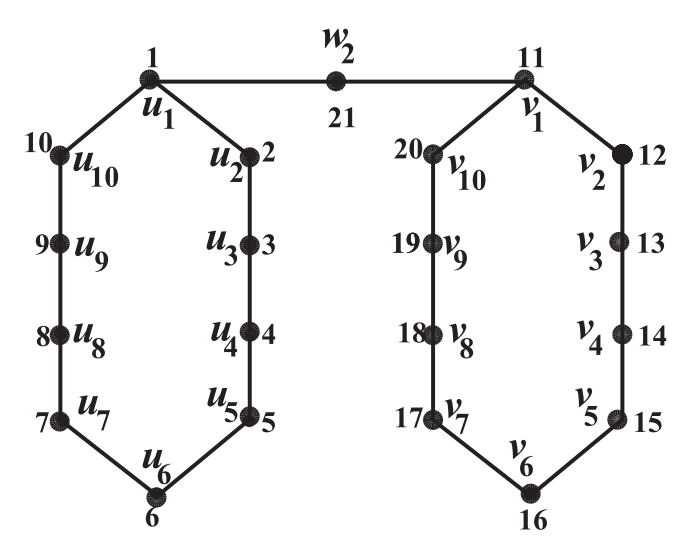

Figure 5. Path joining two copies of $C_{10}$ and Prime labeling 